
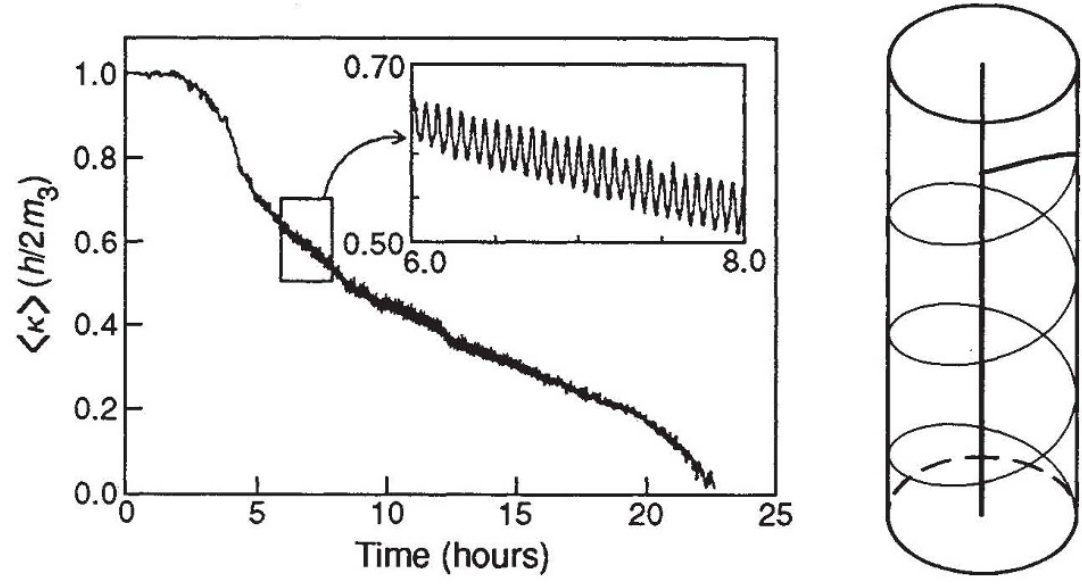

Left, the effective circulation around a wire in superfluid ${ }^{3} \mathrm{He}$, measured as a function of time by Packard and colleagues. The data have been normalized by division by the predicted quantum of circulation $\left(h / 2 m_{3}\right)$. Right, sketch to show how the vortex gradually unzips from the wire, which runs up the axis. The vortex surrounds the wire, from the bottom up to where it has unzipped, and then connects approximately radially to the surrounding brass cylinder. As the radial part of the vortex precesses under the action of hydrodynamic forces it dissipates energy, causing the vortex to shrink downwards along the wire and resulting in a helical motion of the point of attachment to the wall.

imposed on the overall decrease is a periodic oscillation (see inset).

How are these strange results to be explained? The occurrence of circulations apparently equal to non-integral multiples of the expected quantum of circulation is actually not new - something very similar was observed for superfluid ${ }^{4} \mathrm{He}$ by Vinen, who interpreted the effect as an indication that vortices can sometimes surround the wire, not completely as the theory assumes, but over only a fraction of its length. He assumed that the vortex left the wire at some point and that its end was attached (free ends would be unstable and are therefore not allowed) to the inner wall of the container. In Vinen's experiments, however, the measured circulations were stable (unless the wire or apparatus were banged) and did not change with time. Nonetheless, it seems likely that something rather similar is happening in superfluid ${ }^{3} \mathrm{He}$. Packard and colleagues attribute the evident lack of stability to the larger core size of the ${ }^{3} \mathrm{He}$ vortices $\left(1,000\right.$ times larger than those in $\left.{ }^{4} \mathrm{He}\right)$, which means that their ends are much less likely than those of ${ }^{4} \mathrm{He}$ vortices to be stabilized by pinning to microscopic protuberances on the wire or the walls of the cell.

If one thus accepts the notion that an end of the vortex can easily slide over the surface of the wire or walls (and will therefore position itself so as to minimize the free energy), it becomes possible to interpret the results in the figure. Initially, when the rotation first stops, the vortex envelops the whole wire and the theoretically predicted value of $K$ is measured. After a while, however, some kind of random fluctuation occurs (and the geometry of the wire's attachment points will determine how easily it can happen in practice) whereby one end of the vortex becomes separated from the wire and moves instead to the inner wall of the surrounding brass cylinder.

The situation is now radically different. Although the measured value of $K$ is initially still almost equal to the predicted one, because almost the whole of the wire is still enveloped by the vortex, the configuration has become inherently unstable: hydrodynamic forces cause a precession of the element of vortex connecting the wire to the wall, a process that is dissipative because the moving vortex core scatters the quasiparticles (thermal excitations) that are always present in the superfluid at finite temperature. The only place the dissipated energy can come from is the vortex itself, which correspondingly shrinks back gradually along the wire; as it unzips from the wire, its detached end will therefore precess in a helix (see figure). The circulation as measured in the experiment (on the assumption that the vortex surrounds the entire wire) will therefore seem to decrease continuously, finally reaching zero when no vortex remains, exactly as observed.

The authors argue convincingly that the fast periodic oscillation in the figure (inset) corresponds to the precession of the radial element of vortex round and round the cell. The effect arises because (serendipitously?) the wire was positioned slightly off axis in the cell: when the radial section of vortex becomes shorter, the section of vortex enveloping the wire has to lengthen slightly so as to conserve energy, causing the apparent circulation to increase momentarily.

P. V. E. McClintock is in the School of Physics and Materials, University of Lancaster, Lancaster LA1 AYB, UK.

\section{Liquid vision}

WHY are our eyes, those vital sense organs, so imperfect? About half of us need spectacles all the time; by middle age, nearly all of us need them for reading and other close work. Daedalus once advocated wearing an eye-patch from early youth, to save one eye from the fatigue and work-hardening of constant use. In middle age, you could take the patch off, and would then have one youthful eye for reading the small print. He now returns to the field with a subtler ophthalmic strategy.

He has been inspired by the strange medical fact that diabetics sometimes suffer from attacks of long-sightedness. Their sugar concentration is unstable. It is liable to fall, not only in their blood, but also in the vitreous humour of their eyes. Their eyes lose refractive power, making them long-sighted. Drunks also suffer notoriously from focusing problems, an effect which Daedalus similarly blames on the alcohol building up in their eye-fluids.

So DREADCO biochemists are devising a range of optical elixirs and visionpills to adjust or fine tune the consumer's sight. Most chemical substances have a higher refractive index than the eye-fluid, so an elixir of short sight seems fairly feasible. Potassium chloride is already sold as a sodium-free substitute for salt, and Daedalus suggested making this elixir from more exotic and refractive salt analogues such as potassium bromide, rubidium iodide and so on. The DREADCO chemists rapidly overruled this simplistic notion; they are scanning the wider field of organic substances. They plan to synthesize a specialized range of biologically inert but powerfully refractive fluoro-sugars.

DREADCO's 'Bottled Short Sight' will be welcomed by scholars, watchmakers, and others who need to settle to long periods of close work. With luck, it could even last in the body for days. A carefully calibrated daily dose could then provide permanent ophthalmic correction. The happy consumer would at last be free of the curse of spectacles.

A short-lived formulation, rapidly degraded in the body, could provide useful bursts of short sight for entomologists, botanists and interpreters of insurance contracts. It might even be welcomed by lovers, whose vision is exercised at very close range and usually without their glasses. The ability to see one's beloved in sharp focus even in intimate proximity, should powerfully enhance the romantic experience. But less confident lovers may still prefer the traditional alcohol, to defocus their swain's vision and preserve the misty illusion of their fading charms.

David Jones 